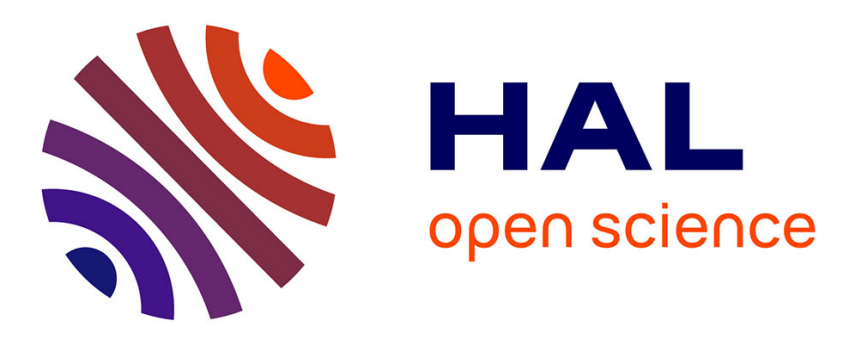

\title{
Elastic-net based beamforming in medical ultrasound imaging
}

Teodora Szasz, Adrian Basarab, Mircea-Florin Vaida, Denis Kouamé

\section{To cite this version:}

Teodora Szasz, Adrian Basarab, Mircea-Florin Vaida, Denis Kouamé. Elastic-net based beamforming in medical ultrasound imaging. 13th IEEE International Symposium on Biomedical Imaging: From Nano to Macro (ISBI 2016), Apr 2016, Prague, Czech Republic. pp. 477-480. hal-01566922

\section{HAL Id: hal-01566922 \\ https://hal.science/hal-01566922}

Submitted on 21 Jul 2017

HAL is a multi-disciplinary open access archive for the deposit and dissemination of scientific research documents, whether they are published or not. The documents may come from teaching and research institutions in France or abroad, or from public or private research centers.
L'archive ouverte pluridisciplinaire HAL, est destinée au dépôt et à la diffusion de documents scientifiques de niveau recherche, publiés ou non, émanant des établissements d'enseignement et de recherche français ou étrangers, des laboratoires publics ou privés. 


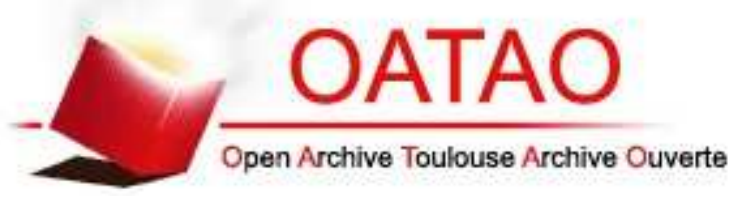

\section{Open Archive TOULOUSE Archive Ouverte (OATAO)}

OATAO is an open access repository that collects the work of Toulouse researchers and makes it freely available over the web where possible.

This is an author-deposited version published in : http://oatao.univ-toulouse.fr/ Eprints ID : 16891

The contribution was presented at ISBI 2016 :

http://biomedicalimaging.org/2016/

To cite this version : Szasz, Teodora and Basarab, Adrian and Vaida, MirceaFlorin and Kouamé, Denis Elastic-net based beamforming in medical ultrasound imaging. (2016) In: 13th IEEE International Symposium on Biomedical Imaging: From Nano to Macro (ISBI 2016), 13 April 2016 - 16 April 2016 (Prague, Czech Republic).

Any correspondence concerning this service should be sent to the repository administrator: staff-oatao@listes-diff.inp-toulouse.fr 


\title{
ELASTIC-NET BASED BEAMFORMING IN MEDICAL ULTRASOUND IMAGING
}

\author{
Teodora Szasz $\quad$ Adrian Basarab ${ }^{\star} \quad$ Mircea-Florin Vaida ${ }^{\dagger} \quad$ Denis Kouamé $^{\star}$ \\ * Université de Toulouse, IRIT, UMR CNRS 5505, France \\ $\dagger$ Technical University of Cluj-Napoca, Romania
}

\begin{abstract}
This paper presents a new way of addressing beamforming in ultrasound imaging, by formulating it, for each image depth, as an inverse problem solved using elastic-net regularization. This approach was evaluated on both simulated and in vivo data showing a gain in contrast, while maintaining an increased value of the signal-to-noise ratio compared to two standard ultrasound beamforming methods.
\end{abstract}

Index Terms - Ultrasound imaging, beamforming, inverse problems, beamspace processing, elastic-net regularization

\section{INTRODUCTION}

Medical ultrasound (US) imaging (or sonography) is one of the most used imaging modalities due to its ease-of-use, low-cost, and non-ionizing characteristics.

Its principle is based on the interaction between the human tissues and ultrasound waves produced by the US probe elements through their piezoelectric capabilities. Standard US probes are containing multiple elements (typically between 16 and 256) that usually can form linear, annular, or circular arrays capable to both transmit the US waves to human body and receive the reflected echoes by the tissues. These echos, transformed into digital signals, represent the raw data acquired by the ultrasound system.

Beamforming (BF) in reception offers the possibility to suppress the signals reflected by the sources in the scanned medium that are interfering with the desired ones. Thus, BF plays a key role in the improvement of the spatial resolution, the signal-to-noise ratio and the contrast of the beamformed image, also called radio-frequency (RF) image which is composed by multiple juxtaposed RF signals. For display purpose, some post-processing techniques like demodulation and log-compression are then applied to the RF signals in order to form the final US image, called B-mode (brightness mode image).

With the classical delay-and-sum (DAS) beamformer, the raw data is firstly focused to compensate the time-of-flight de-

This work has been supported by the CIMI Labex, Toulouse, France, under grant ANR-11-LABX-0040-CIMI within the program ANR-11-IDEX-0002-02. lays, then weighted and summed up. The weighting is done through apodization functions (e.g. the Hanning window) independent on the raw data. The quality in terms of resolution and contrast of DAS beamformer is limited. To improve the quality of the DAS beamformed images, a large variety of adaptive beamformers were proposed in literature, adapting the apodization coefficients to the raw data. The most used adaptive beamformer is minimum variance (MV) beamformers (e.g. [1]) which is inspired from the well known Capon filter. Based on the estimated covariance matrix, MV BF improves the contrast and the spatial resolution of the images, at the cost of increased computational time. Moreover, MV BF raises other problems, related to bad conditioned covariance matrices. Diagonal loading techniques (see e.g., [2]), time and spatial averaging [3], or the iterative adaptive approach (IAA) [4] can be used to overcome these limitations.

Instead of using fixed (DAS) or adaptive (MV) apodization functions, we have recently proposed in [5] a new BF model for medical US imaging that takes into account the positions of the elements and reflectors in order to relate the desired signals to the raw data. This results in an inverse problem which use Laplacian or Gaussian priors through Basis Pursuit (BP), respectively Least Squares methods. We were thus able to obtain two complementary results, one that produced sparse images and one generating smoother results [5]. This formulation also offered the possibility to highly reduce the number of US emissions during the scanning procedure, by integrating it with beamspace processing technique [6].

In this work we propose to further consider a compromise between sparsity and smoothness in the resulted images, by imposing an elastic-net regularization (see e.g. [7] and [8]). The proposed elastic-net (EN) beamformer minimizes a weighted sum of the $\ell_{1}$ and $\ell_{2}$-norms, with respect to the data, therefore combining the advantages offered by the two norms.

\section{BACKGROUND ON US BEAMFORMING}

Without loss of generality, we consider herein an $M$-element US probe, with the pitch (the spacing between elements) equal to $\frac{\lambda}{2}$, where $\lambda=\frac{c}{f_{0}}$, with $c$ denoting the speed of sound in soft tissues, and $f_{0}$ the transducer's center frequency. A series of $K$ focused beams are steered with different incident 
angles, $\theta_{k}, k=1, \cdots K$, and all the elements are used to receive the reflected echoes. After time-of-flight compensation, the raw data from all $K$ directions have the size $M \times N \times K$, where $N$ depends on the axial sampling frequency and the imaging depth.

In this configuration, the DAS BF process that generates one RF line for each steered emission can be expressed as:

$$
\hat{\boldsymbol{s}}_{k}=\boldsymbol{w}^{H} \boldsymbol{y}_{k},
$$

where $\boldsymbol{y}_{k} \in \mathbb{C}^{M \times N}$ is the time-compensated raw data corresponding to the $k$-th emission, $\boldsymbol{w}$ is the apodization function of size $M \times 1$, and $(\cdot)^{H}$ represents the conjugate transpose.

In contrast to DAS, MV BF adaptively calculates the apodization coefficients as:

$$
\boldsymbol{w}_{M V_{k}}=\frac{\boldsymbol{R}_{k}^{-1} \mathbf{1}}{\mathbf{1}^{T} \boldsymbol{R}_{k}^{-1} \mathbf{1}},
$$

where $\boldsymbol{R}_{k}=E\left[\boldsymbol{y}_{k} \boldsymbol{y}_{k}^{H}\right]$ is the covariance matrix of $\boldsymbol{y}_{k}$ and $\mathbf{1}$ is a length $M$ column-vector of ones. These weights are then used to calculate the desired RF beamformed lines following (1).

\section{PROPOSED ELASTIC-NET BASED BEAMFORMING}

\subsection{Direct model formulation}

Contrarily to DAS and MV BF where the raw data is axially beamformed to form the RF signals, the proposed method is processing the raw data laterally, range by range. For a given range $n, n=1, \cdots, N$, the direct model relating the desired signal to the data is given by:

$$
\hat{\boldsymbol{s}}[n]=\left(\boldsymbol{A}^{H} \boldsymbol{A}\right) \boldsymbol{x}[n]+\boldsymbol{g}[n],
$$

where $\hat{\boldsymbol{s}}[n] \in \mathbb{C}^{K \times 1}$ is a lateral scanline extracted from a classically beamformed image (e.g. DAS), $\boldsymbol{A}$ is a classical steering matrix [9], $\boldsymbol{x}[n]$ is the desired signal and $\boldsymbol{g}[n]$ an additive white Gaussian noise. Note that for computational reasons, the model in (3) takes as input the classically beamformed data instead of the raw data (see e.g. [5] and [10]). To decrease the number of US emissions required in BF process, the beamspace processing [11] technique can be applied to (3), thus decreasing the size of the data vector $\hat{s}[n]$ by a factor of $K / P$. After applying such a beamspace technique, the model in (3) can be written as:

$$
\boldsymbol{z}[n]=\boldsymbol{D}^{H} \hat{\boldsymbol{s}}[n]=\left(\boldsymbol{A}_{B S}^{H} \boldsymbol{A}\right) \boldsymbol{x}[n]+\boldsymbol{D}^{H} \boldsymbol{g}[n],
$$

where $D$ of size $K \times P$ is the beamspace decimation matrix, $\boldsymbol{A}_{B S}^{H}$ of size $P \times M$ is the beamspaced steering matrix, and $\boldsymbol{x}[n]$ of size $K \times 1$ is the lateral profile at range $n$ to be estimated. The model in (4) can be furthered considered as an inverse problem, where $\boldsymbol{z}[n]$ is the decimated DAS beamformed data obtained for $P<K$ emissions (the observations) and $\boldsymbol{x}[n]$ the desired lateral profile at depth $n$.

\subsection{Model inversion}

In this paper, we propose to solve the ill-posed inverse problem in (4) using the following regularization referred to as elastic-net $(\mathrm{EN})$ regularization:

$$
\begin{aligned}
\boldsymbol{x}_{E N}[n] & =\underset{\boldsymbol{x}[n]}{\operatorname{argmin}}\left(\left\|\boldsymbol{z}[n]-\left(\boldsymbol{A}_{B S}^{T} \boldsymbol{A}\right) \boldsymbol{x}[n]\right\|_{2}^{2}\right. \\
& \left.+\gamma\left(\|\boldsymbol{x}[n]\|_{2}^{2}+\epsilon\|\boldsymbol{x}[n]\|_{1}\right)\right),
\end{aligned}
$$

where $\|\cdot\|_{1}$ and $\|\cdot\|_{2}^{2}$ denote the $\ell_{1}$, respectively $\ell_{2}$-norms. In our experiments, in order to decrease the number of hyperparameters to tune, we considered $\gamma \epsilon=(1-\gamma)$, turning the regularization term in (5) into:

$$
\operatorname{pen}(\boldsymbol{x}[n])=\gamma\|\boldsymbol{x}[n]\|_{2}^{2}+(1-\gamma)\|\boldsymbol{x}[n]\|_{1},
$$

where $\operatorname{pen}(\boldsymbol{x}[n])$ is the penalization term that influences the sparsity of the result. Thus, for $\gamma=0$ the problem turns into a basis pursuit algorithm, while when $\gamma=1$ we obtain the Tikhonov regularization. In the following, let us denote by $\boldsymbol{\Psi} \in \mathbb{C}^{P \times K}$ the measurement matrix formed as $\boldsymbol{\Psi}=\boldsymbol{A}_{B S}^{T} \boldsymbol{A}$. Moreover, we define by $S_{\gamma}$ the soft thresholding operator, having the following function for any $\gamma>0$ :

$$
S_{\gamma}(t)= \begin{cases}t-\frac{\gamma}{2}, & \text { if } \mathrm{t}<\frac{\gamma}{2} \\ 0, & \text { if }|t| \leq \frac{\gamma}{2} \\ t+\frac{\gamma}{2}, & \text { if } \mathrm{t}<-\frac{\gamma}{2}\end{cases}
$$

Algorithm 1, based on [12], describes the main steps used to minimize the function in (5). We remind that this optimization is done independently, range by range, resulting into the consecutive lateral profiles of the final beamformed image.

$$
\begin{aligned}
& \text { Input: } \gamma, \boldsymbol{z}[n], \boldsymbol{\Psi} \\
& \text { Output: } \boldsymbol{x}^{p}[n] \\
& \text { initialization: } \boldsymbol{x}^{0}[n]=\mathbf{0} \\
& \text { // damping factor: } \\
& \tau=\frac{1}{2-\gamma} \\
& / / \text { fix the step size using the matrix 2-norm of } \boldsymbol{\Psi} \boldsymbol{\Psi}^{T} \text { : } \\
& \delta=\frac{1}{\text { normest }\left(\boldsymbol{\Psi} \boldsymbol{\Psi}^{T}\right) \times 1.1} \\
& \text { while convergence not reached do } \\
& \quad \begin{array}{l}
p:=p+1 ; \\
\boldsymbol{x}^{p}[n]= \\
\tau S_{\gamma K \delta}\left(\boldsymbol{x}^{p-1}[n]+\delta \boldsymbol{\Psi}^{T}\left(\boldsymbol{z}[n]-\boldsymbol{\Psi} \boldsymbol{x}^{p-1}[n]\right) ;\right.
\end{array} \\
& \text { end }
\end{aligned}
$$

Algorithm 1: Elastic-net beamforming of one lateral profile via Iterative Soft Thresholding.

\section{RESULTS AND DISCUSSION}

To evaluate the proposed method, we considered two cases: one that contains simulated data of 8 point reflectors arranged between $35 \mathrm{~mm}$ and $55 \mathrm{~mm}$ in depth and an experiment that 

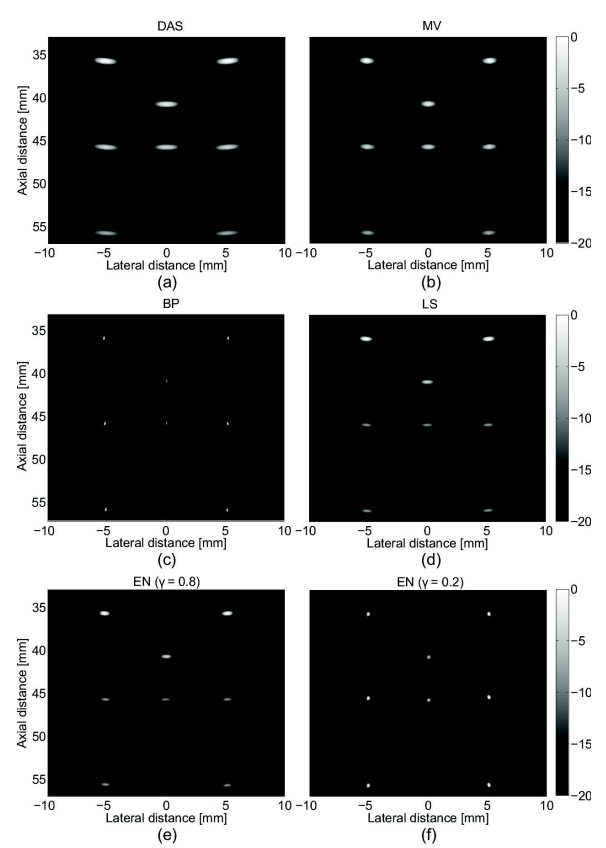

Fig. 1. (a) DAS, (b) MV, (c) BP, (d) LS, (e) EN ( $\gamma=0.8)$, and (f) $\mathrm{EN}(\gamma=0.2) \mathrm{BF}$ results of a sparse medium.

uses in vivo data of a healthy thyroid. We compared our method with four other BF methods (DAS using Hanning apodization window, MV [2], BP and LS [5]) in the case of sparse medium, and with DAS, BP, and LS in the case of in vivo data.

\subsection{Simulation results}

The simulation containing the sparse medium was done using the Field II program [13], aiming to evaluate the BF lateral resolution of the proposed method. A 64-element US probe was used for both transmission and reception, with the center frequency, $f_{0}=4 \mathrm{MHz}$ and $96 \%$ of relative bandwidth. The transducer had the following element characteristics: the pitch of $231 \mu \mathrm{m}$, the kerf of $38.5 \mu \mathrm{m}$, and the height of $14 \mathrm{~mm}$. An excitation pulse of two-cycle sinusoidal at $f_{0}$ was used. $K=260$ emissions were steered with angles between $-30 \mathrm{deg}$ and $30 \mathrm{deg}$ in the case of DAS and MV BF, and $P=\frac{260}{5}=52$ emissions in the case of BP, LS, and EN BF methods. The beamformed images are depicted in the Fig. 1. DAS BF (Fig. 1(a)) results in low lateral resolution, further improved by MV BF (Fig. 1(b)). However, the best resolution of the target points is offered by the BP BF (Fig. 1(c)) that perfectly detects the 8 reflectors. This observation is in coherence with the fact that BP privileges sparsity in the results, through the Laplacian statistics. LS BF (Fig. 1(d)) results in smoother solutions compared with BP $\mathrm{BF}$, but with better lateral resolution than MV. As expected, EN with $\gamma=0.8$ (Fig. 1(e)) produces results similar with LS

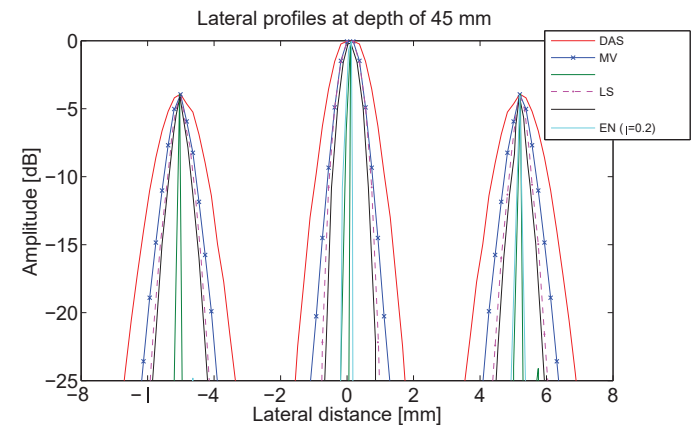

Fig. 2. Lateral profiles of Fig. 1 at depth $45 \mathrm{~mm}$.
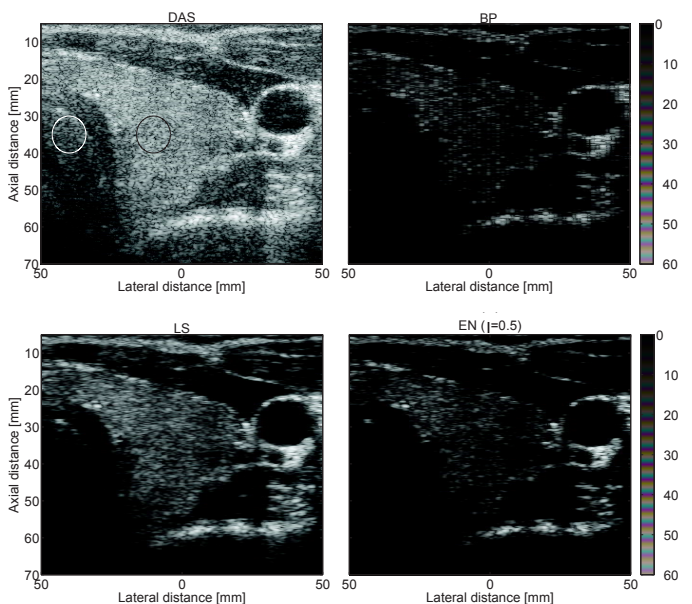

Fig. 3. (a) DAS, (b) BP, (c) LS, and (d) EN $(\gamma=0.8) \mathrm{BF}$ results of in vivo data.

$\mathrm{BF}$, since the hyperparameter $\gamma$ decides the level of smoothness of the solutions, weighting the term related to $\ell_{2}$-norm. Contrarily, EN with $\gamma=0.2$ (Fig. 1(e)) tends to the solution of BP BF. Note that for each simulation, $\gamma$ has been set empirically to its best value.

The remarks above are confirmed by the Fig. 2 showing the lateral variation of the beamformed responses at depth 45 $\mathrm{mm}$. BP BF have the narrower mainlobes, offering the best delimitation of the points. However, the two cases of EN BF are presenting results that are varying between BP and LS, but with better spatial resolution of the target points than DAS and MV.

\subsection{In vivo results}

The data was acquired with the clinical Sonoline Elegra ultrasound system, by using the 7.5L40 P/N 526028-L0850 linear array transducer of Siemens Medical Systems.

The beamformed images using in vivo thyroid data are presented in the Fig. 3. Three image quality metrics were considered, computed on the envelope-detected signals: the 
Table 1. CR, CNR, and SNR values for the in vivo thyroid beamformed images in Fig. 3

\begin{tabular}{llll}
\hline BF Method & CR[dB] & CNR & SNR \\
\hline \hline DAS & 12.4871 & 0.5546 & 0.2155 \\
\hline BP & 20.4878 & 1.1304 & 0.3161 \\
\hline LS & 16.9313 & $\mathbf{1 . 5 6 4 3}$ & $\mathbf{0 . 6 4 1 4}$ \\
\hline EN $(\gamma=0.5)$ & $\mathbf{2 2 . 4 4 8 9}$ & 1.4846 & 0.4571 \\
\hline
\end{tabular}

contrast ratio (CR), the contrast-to-noise ratio (CNR), and the signal-to-noise ratio (SNR). Two regions, $R_{1}$ (the white circle depicted in the Fig. 1(a)) and $R_{2}$ (the black circle depicted in the Fig. 1(b)) were selected for calculating these metrics. CR is defined as [14] $C R=\left|\mu_{R_{1}}-\mu_{R_{2}}\right|$, where $\mu_{R_{1}}$ and $\mu_{R_{2}}$ are the mean values in the region $R_{1}$, respectively $R_{2}$. CNR is defined as [15] $C N R=\frac{\left|\mu_{R_{1}}-\mu_{R_{2}}\right|}{\sqrt{\sigma_{R_{1}}^{2}+\sigma_{R_{2}}^{2}}}$, where $\sigma_{R_{1}}$ and $\sigma_{R_{2}}$ are the standard deviations of intensities in $R_{1}$, respectively $R_{2}$. The SNR was defined as the ratio between the mean value $\mu$ and the standard deviation $\sigma$ in the homogeneous region $R_{2}$ [4]: $S N R=\frac{\mu}{\sigma}$. The obtained values of CR, CNR, and SNR are depicted in the Table 1.

In Fig. 3(a), when using DAS BF the thyroid structure can be hardly distinguished, the contrast in the image being relatively low. Fig. 3(b) shows the result of the BP BF which selects the echoic structures of the image, thus eliminating most of the speckle in the image. Even if the CR and the CNR values are improved compared with DAS, the value of SNR is however comparable to DAS. As expected, LS results in more regular image at the price of lower contrast compared to BP, see Fig. 3(c). The best contrast of the beamformed image while maintaining a relatively high level of SNR and CNR is obtained with EN BF, see Fig. 3(d). In this case, the value of $C R$ is increased by $10 \mathrm{~dB}$ compared with DAS, by about $2 \mathrm{~dB}$ compared with $\mathrm{BP}$ and by $5 \mathrm{~dB}$ compared with LS, see Table 1.

\section{CONCLUSION}

In this paper, we modeled US beamforming as an inverse problem regularized using elastic-net regularization. This formulation offers the possibility to integrate in the BF process the advantages of both sparse and smooth solutions. We have shown that our approach applied to simulated data provides solutions with improved lateral resolution compared to DAS and MV. Evaluated on in vivo data of a thyroid, the elastic-net based method improved the contrast of the image compared with DAS, BP, and LS methods. As a perspective we can consider generalized Gaussian priors that result in $\ell_{p}$-norm min- imization together with taking into account the system point spread function.

\section{REFERENCES}

[1] J.-F. Synnevg, A. Austeng, and S. Holm, "Benefits of minimumvariance beamforming in medical ultrasound imaging," IEEE Transactions on Ultrasonics, Ferroelectrics, and Frequency Control, vol. 56, no. 9, pp. 1868-1879, Sept. 2009.

[2] B.M. Asl and A. Mahloojifar, "Minimum variance beamforming combined with adaptive coherence weighting applied to medical ultrasound imaging," IEEE Transactions on Ultrasonics, Ferroelectrics, and Frequency Control, vol. 56, no. 9, pp. 1923-1931, Sept. 2009.

[3] I.K. Holfort, F. Gran, and J.A. Jensen, "Broadband minimum variance beamforming for ultrasound imaging," IEEE Transactions on Ultrasonics, Ferroelectrics, and Frequency Control, vol. 56, no. 2, pp. 314-325, Feb. 2009.

[4] A.C. Jensen and A. Austeng, "The iterative adaptive approach in medical ultrasound imaging," IEEE Transactions on Ultrasonics, Ferroelectrics, and Frequency Control, vol. 61, no. 10, pp. 1688-1697, Oct. 2014.

[5] Teodora Szasz, Adrian Basarab, and Denis Kouamé, "Beamforming through regularized inverse problems in ultrasound medical imaging," arXiv:1507.08184 [cs], July 2015, arXiv: 1507.08184.

[6] J.J. Fuchs, "Linear programming in spectral estimation. Application to array processing," in , 1996 IEEE International Conference on Acoustics, Speech, and Signal Processing, 1996. ICASSP-96. Conference Proceedings, May 1996, vol. 6, pp. 3161-3164 vol. 6.

[7] Hui Zou and Trevor Hastie, "Regularization and variable selection via the elastic net," Journal of the Royal Statistical Society: Series B (Statistical Methodology), vol. 67, no. 2, pp. 301-320, Apr. 2005.

[8] Christine De Mol, Ernesto De Vito, and Lorenzo Rosasco, "Elastic-net regularization in learning theory," Journal of Complexity, vol. 25, no. 2, pp. 201-230, Apr. 2009.

[9] G.M. Kautz and M.D. Zoltowski, "Beamspace DOA estimation featuring multirate eigenvector processing," IEEE Transactions on Signal Processing, vol. 44, no. 7, pp. 1765-1778, July 1996.

[10] D. Malioutov, M. Cetin, and A.S. Willsky, "A sparse signal reconstruction perspective for source localization with sensor arrays," IEEE Transactions on Signal Processing, vol. 53, no. 8, pp. 3010-3022, Aug. 2005.

[11] Zhi Tian and H.L. Van Trees, "Beamspace MODE," in Conference Record of the Thirty-Fifth Asilomar Conference on Signals, Systems and Computers, 2001, Nov. 2001, vol. 2, pp. 926-930 vol.2.

[12] Sofia Mosci, Lorenzo Rosasco, Matteo Santoro, Alessandro Verri, and Silvia Villa, "Solving Structured Sparsity Regularization with Proximal Methods," in Machine Learning and Knowledge Discovery in Databases, Jos Luis Balczar, Francesco Bonchi, Aristides Gionis, and Michle Sebag, Eds., number 6322 in Lecture Notes in Computer Science, pp. 418-433. Springer Berlin Heidelberg, Sept. 2010, DOI: 10.1007/978-3-642-15883-4_27.

[13] J.A. Jensen and N.B. Svendsen, "Calculation of pressure fields from arbitrarily shaped, apodized, and excited ultrasound transducers," IEEE Transactions on Ultrasonics, Ferroelectrics, and Frequency Control, vol. 39, no. 2, pp. 262-267, Mar. 1992.

[14] Mengling Xu, Xin Yang, Mingyue Ding, and Ming Yuchi, "Spatiotemporally smoothed coherence factor for ultrasound imaging [Correspondence]," IEEE Transactions on Ultrasonics, Ferroelectrics, and Frequency Control, vol. 61, no. 1, pp. 182-190, Jan. 2014.

[15] O.M.H. Rindal, J.P. Asen, S. Holm, and A. Austeng, "Understanding contrast improvements from capon beamforming," in Ultrasonics Symposium (IUS), 2014 IEEE International, Sept. 2014, pp. 1694-1697. 